\title{
IRRATIONAL TRANSFORMATIONS OF THE GENERAL ELLIPTIC ELEMENT.
}

BY PROFESSOR F. H. SAFFORD.

(Read before the American Mathematical Society, April 28, 1917.)

IN 1865 G. G. A. Biermann published as derived from Weierstrass's lectures the following formula:

(1) $\quad F(x)=x_{0}+$

$$
\frac{\sqrt{R\left(x_{0}\right)} \sqrt{S}+\frac{1}{2} R^{\prime}\left(x_{0}\right)\left[s-\frac{1}{24} R^{\prime \prime}\left(x_{0}\right)\right]+\frac{1}{24} R\left(x_{0}\right) R^{\prime \prime \prime}\left(x_{0}\right)}{2\left[s-\frac{1}{24} R^{\prime \prime}\left(x_{0}\right)\right]^{2}-\frac{1}{2} A \cdot R\left(x_{0}\right)} .
$$

$F$ is the solution of

(2) $\left(F^{\prime}\right)^{2}=A F^{4}+4 B F^{3}+6 C F^{2}+4 B^{\prime} F+A^{\prime}=R(F)$.

The accents used with $F$ and $R$ denote differentiation, $x_{0}$ is an arbitrary constant, and $A, B, C, B^{\prime}, A^{\prime}$ are constant coeffcients. Also

$$
\begin{aligned}
& S=4 s^{3}-g_{2} s-g_{3}=4\left(s-\epsilon_{1}\right)\left(s-\epsilon_{2}\right)\left(s-\epsilon_{3}\right), \\
& s=\wp(x), \quad g_{2}=A A^{\prime}+3 C^{2}-4 B B^{\prime}, \\
& g_{3}=A C A^{\prime}+2 B C B^{\prime}-A B^{\prime 2}-A^{\prime} B^{2}-C^{3} .
\end{aligned}
$$

In Enneper, Elliptische Functionen, Greenhill, Elliptic Functions; and Haentzschel, Reduction der Potentialgleichung, are several applications of this formula.

Replacing $x$ by $t+i u$ and $F$ by $x+i y$ in the preceding equations gives

$$
x+i y=\varphi(t+i u),
$$

from which may be obtained in the usual manner two mutually orthogonal families of curves having $t$ and $u$ as parameters. For several cases this has been done by Haentzschel, and the writer has given discussions and extensions in Archiv der Mathematik und Physik. It is evident that (1) becomes very simple when $x_{0}$ is a root of $R(x)=0$, for which Haentzschel's 
results may be consulted. When $x_{0}$ is infinite Enneper obtains from (1)

$$
v=\frac{\sqrt{A} \sqrt{S}+2 B s-B C+A B^{\prime}}{2\left(B^{2}-A C\right)-2 A s},
$$

but does not develop the results. However, the rationalization of (5) gives

(6) $s^{2}-s\left(A v^{2}+2 B v+C\right)+\left(B^{2}-A C\right) v^{2}$

$$
+\left(B C-A B^{\prime}\right) v+\left(C^{2}-A A^{\prime}\right) / 4=0 .
$$

Next placing $x$ equal to 0 in (1),

$$
\begin{aligned}
& s^{2} v^{2}-s\left(A^{\prime}+2 B^{\prime} v+C v^{2}\right)+\left(B^{\prime 2}-A^{\prime} C\right) \\
& +\left(B^{\prime} C-A^{\prime} B\right) v+\left(C^{2}-A A^{\prime}\right) v^{2} / 4=0
\end{aligned}
$$

which becomes (6) when $v$ is replaced by $1 / v$, noticing the interchange of constants required by (2). Later in this paper reference will be made to (7) in another discussion.

In using (1) to obtain orthogonal curves it is of great advantage to make the following changes: $F(x)$ is replaced by $x_{0}+v$ and (2) is written

whence

$$
\begin{gathered}
\left(v^{\prime}\right)^{2}=a v^{4}+4 b v^{3}+6 c v^{2}+4 d v+e=R\left(x_{0}+v\right) \\
{\left[v=x^{\prime}+i y^{\prime}\right],}
\end{gathered}
$$

(9) $\quad a=\frac{R^{\prime \prime \prime \prime}\left(x_{0}\right)}{4 !}=A, \quad 4 b=\frac{R^{\prime \prime \prime}\left(x_{0}\right)}{3 !}, \quad 6 c=\frac{R^{\prime \prime}\left(x_{0}\right)}{2 !}$,

$$
4 d=R^{\prime}\left(x_{0}\right), \quad e=R\left(x_{0}\right),
$$

$$
g_{2}=a e-4 b d+3 c^{2}, \quad g_{3}=a c e+2 b c d-a d^{2}-e b^{2}-c^{3} .
$$

The rationalized form of (1) thus becomes

(10) $s^{2} v^{2}-s\left(c v^{2}+2 d v+e\right)+\left(d^{2}-c e\right)+(c d-b e) v$

which is of the same form as (7).

$$
+\left(c^{2}-a e\right) v^{2} / 4=0,
$$

Greenhill, on page 151 (l. c.), refers to (1) as a method of reducing the general elliptic element to the Weierstrass form. Now in using (1) as a solution of (2) it is required that $s$ shall satisfy

$$
\left(s^{\prime}\right)^{2}=4 s^{3}-g_{2} s-g_{3},
$$


so that one value is of course $8(x)$. Since (11) is only a special case of (2) the corresponding form of (1) gives the solution

$$
\bar{s}=\frac{\sqrt{\left(-g_{3}\right)} \sqrt{S}-\frac{1}{2} g_{2} s-g_{3}}{2 s^{2}}
$$

or in rational form,

$$
s^{2} \bar{s}^{2}+g_{2} s s / 2+g_{3}(s+\bar{s})+g_{2}^{2} / 16=0 .
$$

By elimination of $s$ between (10) and (13) there is obtained a new equation involving $v$ and $\bar{s}$ which is a still more complicated solution of (8), or in other words a method of reducing the general elliptic element to the Weierstrass form. Because $v$ and $\bar{s}$ do not enter symmetrically this equation is too complicated to be of interest.

The conjugate of (10) formed by changing $s$ and $v$ into $\sigma$ and $w$ respectively provides a transformation of the general into the Weierstrass form and vice versa, so that making $s$ and $\sigma$ identical, and then eliminating $s$ gives an identical transformation of the general elliptic element. After cancellation of the trivial factor $(v-w)^{2}$ the result is

$$
\begin{aligned}
v^{2} w^{2}\left(b^{2} e^{2}-a e d^{2}\right)+v w(v+w)\left(3 b c e^{2}-2 b e d^{2}-a d e^{2}\right) \\
+v w\left(\frac{9 c^{2} e^{2}}{2}-2 b d e^{2}-2 d^{4}-\frac{a e^{3}}{2}\right) \\
+(v+w)\left(3 c d e^{2}-b e^{3}-2 d^{3} e\right) \\
+\left(v^{2}+w^{2}\right)\left(\frac{9 c^{2} e^{2}}{4}-3 c d^{2} e+d^{4}-\frac{a e^{3}}{4}\right)=0 .
\end{aligned}
$$

If $s$ and $\sigma$ in (10) and its conjugate are written $8(t+i u)$ and $\wp(t-i u)$ respectively, and $u$ is eliminated, the resulting equation of the sixteenth degree is resolvable into one factor of the eighth degree and two equal factors each of the fourth degree having $\wp(2 t)$ as a parameter. Now if $\wp(2 t)$ is taken as $\left(d^{2}-c e\right) / e$ the equal factors are identical with (14), which also, as is evident from the preceding, corresponds with $s-\sigma=0$. Lastly the discriminant of (14) as a quadratic equation in $v$ is

$$
\begin{aligned}
& e\left(a w^{4}+4 b w^{3}+6 c w^{2}+\right.4 d w+e)\left(d^{2}-c e-e \epsilon_{1}\right) \\
& \times\left(d^{2}-c e-e \epsilon_{2}\right)\left(d^{2}-c e-e \epsilon_{3}\right) .
\end{aligned}
$$

University of Pennsylvania, April, 1917. 